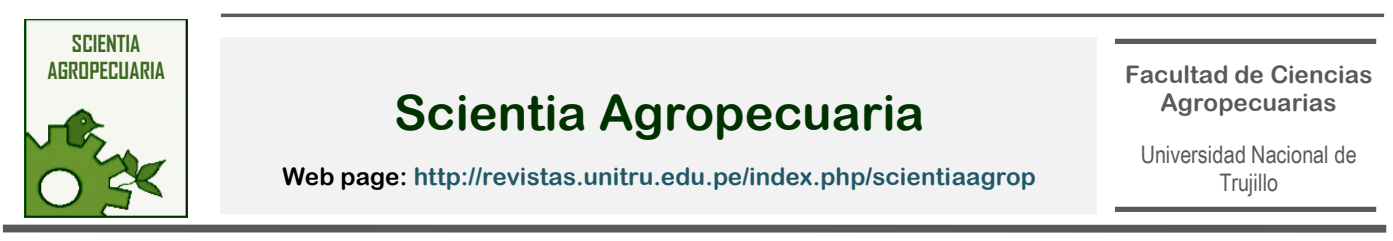

\title{
REVIEW
}

\section{Climate change in the Andes and its impact on agriculture: a systematic review}

\section{El cambio climático en los andes y su impacto en la agricultura: una revisión sistemática}

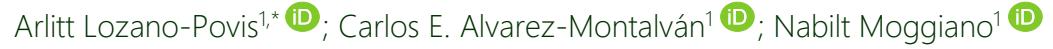 \\ Universidad Continental, Campus Huancayo, Av. San Carlos 1980, Urb. San Antonio, Huancayo, Junín. Peru.
}

*Corresponding author: 70041324@continental.edu.pe (A. A. Lozano-Povis).

Received: 17 September 2020. Accepted: 25 February 2021. Published: 10 March 2021.

\begin{abstract}
In recent years, agriculture in the Andes has shown greater sensitivity to climate change, favoring processes of soil erosion, retreat of glaciers, loss of vegetation cover, increased intensity of rainfall and alteration in the dynamics of crops in the region such as: potato, quinoa, corn, among others. This motivated many authors to develop regional model simulations to estimate the vulnerability index of these agricultural systems to these climatic events, allowing them to provide a more reliable climatological data in the presence of hot and dry winds. In this review article, the main contributions provided by various researchers regarding the impact of climate change on Andean agriculture are detailed. According to the collected information, it is concluded that climate change in the Andes will cause countries such as Brazil, Bolivia, Ecuador, Venezuela, Guyana and Colombia, to increase their local temperature, potential for evapotranspiration and water scarcity, causing the loss of important crops such as rice. In contrast, countries such as Peru, Argentina, Chile, Bolivia and Uruguay will register lower temperatures that will affect their production and yield in crops such as quinoa, potatoes, tarwi, among others.
\end{abstract}

Keywords: agricultural systems; vulnerability; glaciers; erosion; climate model; hydrological service.

\section{Resumen}

En los últimos años, la agricultura de los Andes ha mostrado mayor sensibilidad al cambio climático, favoreciendo procesos de erosión del suelo, retroceso de glaciares, pérdida de cobertura vegetal, incremento en la intensidad de lluvias y alteración en la dinámica de los cultivos de la región como: papa, quinua, maíz, entre otros. Lo que motivó que, muchos autores desarrollen simulaciones de modelos regionales para estimar el índice de vulnerabilidad de estos sistemas agrícolas ante estos eventos climáticos, permitiéndoles otorgar una data climatológica más confiable en presencia de vientos cálidos y secos. En el presente artículo de revisión, se detallan los principales aportes brindados por diversos investigadores respecto al impacto del cambio climático en la agricultura andina. Según los antecedentes recopilados se concluye que, el cambio climático en los Andes provocará que países como Brasil, Bolivia, Ecuador, Venezuela, Guyana y Colombia, incrementen su temperatura local, potencial de evapotranspiración y escasez de agua, ocasionando la pérdida de cultivos importantes como el arroz. En contraste, países como Perú, Argentina, Chile, Bolivia y Uruguay registrarán temperaturas más bajas que afectarán su producción y rendimiento en cultivos como la quinua, papa, tarwi, entre otros.

Palabras clave: sistemas agrícolas; vulnerabilidad; glaciares; erosión; modelo climático; servicio hidrológico.

DOI: https://dx.doi.org/10.17268/sci.agropecu.2021.012

Cite this article:

Lozano-Povis, A., Alvarez-Montalván. C. E., \& Moggiano-Aburto, N. (2021). El cambio climático en los andes y su impacto en la agricultura: una revisión sistemática. Scientia Agropecuaria, 12(1), 101-108.

\section{Introducción}

Los últimos años han sido testigos de los numerosos esfuerzos realizados para comprender y soportar la complejidad ecológica de los ecosistemas que permiten la provisión de bienes y servicios. Por lo que resulta importante tener en cuenta las dimensiones humanas del cambio climático, aspecto que va tomando mayor relevancia en la comunidad científica (Scoville, 2018). El efecto más notorio de que las temperaturas medias en los Andes de América del Sur se están incrementando es el retroceso acelerado de los glaciares, que afecta directamente los sistemas agrícolas presentes en la zona (Ponce, 2020). A continuación, se presenta la Figura 1, elaborada en ArcGis versión 10.5, en el que se identifican los puntos más importantes en lo que respecta a cuerpos de agua, según la proyección realizada por la organización Climate Impact Lab al 2050 de la variabilidad climática por cada país. Además de las principales zonas agrícolas identificadas por la Organización de las Naciones Unidas para la Alimentación y la Agricultura (FAO), cuerpos de agua y glaciares presentes en los Andes; en suma, indican que el efecto del cambio climático será significativo en unos años en países como Brasil, Bolivia, Ecuador, Venezuela, Guyana y Colombia. A diferencia de Perú, Argentina, Chile y Uruguay, donde las temperaturas se tornarían más bajas. 

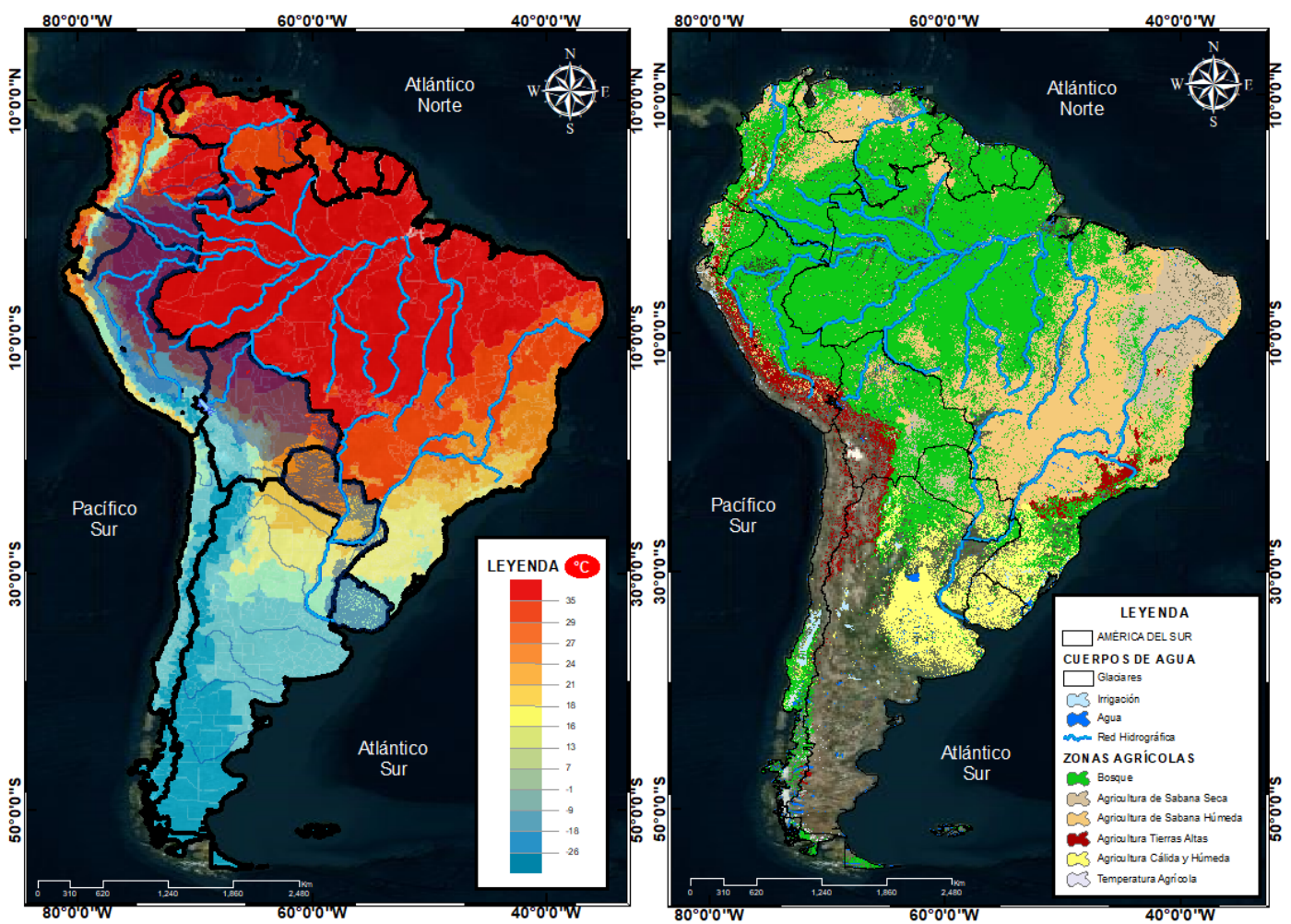

Figura 1. Mapa de los principales cuerpos de agua, zonas agrícolas y cambio climático en América del Sur.

Ambos escenarios permiten afirmar que, los servicios hidrológicos disminuirán su capacidad de abastecimiento en las comunidades altoandinas, particularmente en regiones pobladas donde este cambio será a condiciones climáticas más secas. Además, los hogares rurales empobrecidos (como la mayoría de los pequeños agricultores andinos) son altamente vulnerables al clima (Berrouet et al., 2020) y la respuesta biológica del rendimiento de los cultivos será deficiente o casi nula. Más importante aún, el cambio climático alterará la forma en que las especies interactúan entre sí y con su entorno. Dicha interrupción conduce a la pérdida o deterioro de los servicios y la funcionalidad de los ecosistemas, lo que en última instancia compromete el bienestar humano (Ríos et al., 2018). Es así como, en el presente artículo de revisión se pretende detallar los diferentes aportes brindados por investigadores respecto al impacto del cambio climático en la agricultura andina, con el fin de explicar los sucesos que podrían haber ocurrido en el transcurso de la historia del planeta y predecir sus efectos futuros.

\section{Cambio climático durante las eras geológicas en los Andes}

En los últimos 50 años, el clima de los Andes tropicales tuvo una variación de $0,1^{\circ} \mathrm{C} /$ década lo cual ha influido en el comportamiento de los glaciares alterando su balance energético y favoreciendo su pérdida por escorrentía. Por otro lado, los lagos que están dentro de los valles generalmente están compuestos por lechos rocosos e hipsometrías similares, aunque con distintas elevaciones y que se relacionan con el número de glaciaciones pasadas ocurridas en estas zonas (Stansell et al., 2014).
Tanto la vegetación y la fuerza de los vientos permitieron el restablecimiento de la historia del paleoclima (Jara et al., 2019) mediante restos de polen y carbón vegetal que, sirvieron como indicadores para estimar la presencia e incremento de bosques durante el Holoceno, demostrando su adaptabilidad al cambio climático y los regímenes de desorganización natural. Así mismo, durante el Monzón de Verano de América del Sur, sucesos como la deforestación e incendios forestales, no afectaron los bosques quedando evidenciados en registros paleoclimatológicos (Viveen et al., 2019) e indicando que desde hace 12.000 años los ríos de los Andes peruanos son sensibles al cambio climático.

Pasando a la época del cuaternario los principales movimientos de masas se agruparon en dos períodos de tiempo que corresponderían a períodos húmedos asociados al incremento de la precipitación y la temperatura (Trauth et al., 2003). En la Tabla 1 se muestran investigaciones de los últimos 4 períodos geológicos clasificados según las variables climáticas y cuerpos de agua afectados por el cambio climático.

La variación climática, producto de incendios registrados cerca de glaciares en Bolivia, provocó que muchos de estos bosques limiten su expansión perjudicando el vínculo existente entre la vegetación y los impulsores climáticos como: nubosidad, temperatura, viento, etc. (Williams et al., 2011), evidenciando que las actividades humanas no causaron impacto alguno en el paisaje local. Respecto a la composición de isótopos de carbono de hace 4300 años, permitieron establecer que se registraron cambios importantes del clima en la Cordillera Occidental de los Andes del sur del Perú (Engel et al., 2014) 
propiciando que climas cálidos pasen a ser extremadamente secos. Asimismo, en el último período glacial se repitieron estos patrones a escala mundial y (Fritz et al., 2010) analizaron y registraron los sedimentos encontrados en el lago Titicaca cuyas propiedades geoquímicas eran controladas por la abundancia relativa de componentes terrígenos y que probablemente se formaron en climas húmedos, mientras que sedimentos de carácter pelágico en climas secos.

Finalmente, llegamos al período del antropoceno donde se añade el término de agrobiodiversidad para comprender el mecanismo de interacción entre el ser humano, medio ambiente, impacto de cambio climático y la contaminación; aspectos que se tradujeron en sequías interanuales prolongadas, aumento de la variación intraanual de las precipitaciones y que representan grandes desafíos en la agricultura, biodiversidad, sociedad y salud (Zimmerer et al., 2019).

\section{Vulnerabilidad de especies al cambio climático}

En general, la biodiversidad es la encargada de sustentar funciones y servicios esenciales para la agricultura (Ortiz et al., 2021) ha sufrido efectos directos e indirectos de la elevación, temperatura, precipitación, alteración de la estructura de la vegetación y el cambio climático (Santillán et al., 2020) por lo que está continuamente amenazada por actividades antropogénicas, hecho que motivó que (Ramirez et al., 2014) utilicen modelos de distribución de especies para evaluar sus posibles cambios futuros y estimar el tamaño del nicho climático. En total analizaron 11012 especies de aves y plantas vasculares proyectando que, para la década del 2050, más del 50\% de éstas sufrirán reducciones de almenos el $45 \%$ en su nicho climático, mientras que el $10 \%$ podrían estar extintas.

Este tema se ha convertido en un campo ampliamente investigado en los últimos años, se publicaron al menos 743 artículos que informan sobre este tema, así como la conservación y la prevalencia de los métodos de evaluación mecanicista, especialmente en la investigación centrada en plantas (Ríos et al., 2018). Algunas de las investigaciones más influyentes se muestran en la Tabla 2.

Por ello, la identificación de estas especies resulta importante, ya que a pesar de que actualmente no están amenazadas es probable que lo estén en el futuro, según se intensifiquen los impactos del cambio climático (Godefroid \& Vanderborght, 2010), requiriendo para su evaluación los índices de vulnerabilidad indicados en la literatura o en la web (Tuberville et al., 2015). Además, los pastizales altoandinos tropicales (páramos y punas) y los bosques montanos, experimentarán cambios negativos en la riqueza de especies y altas tasas de rotación.

En el caso de las especies de vida más larga, como árboles y arbustos, tienen menor capacidad de recombinación genética y menos oportunidades de acumular mutaciones potencialmente beneficiosas, particularmente cuando el tamaño efectivo de la población es bajo, lo que puede influir en su capacidad de adaptación (Butt \& Gallagher, 2018). Por lo que, adaptarse a estos impactos requeriría un enfoque de conservación basada en redes de paisajes, incluidas las áreas protegidas, zonas de amortiguamiento y corredores (Agudelo et al., 2019).

\section{Impacto del Cambio Climático en la agricultura 4.1 Efectos en la agricultura de la zona andina}

La disminución de la producción agrícola por el cambio climático en algunas zonas de los Andes ha provocado el incremento de procesos migratorios de muchas familias en búsqueda de mejores oportunidades. Si bien este proceso satisface sus necesidades de consumo, reduce la capacidad de mano de obra, lo que puede afectar negativamente las cosechas futuras en cantidad y diversidad del consumo de alimentos (Blackmore et al., 2021).

Además, la diversidad de regiones agroclimáticas en los Andes, geografías desafiantes, economías en crecimiento, diversos sistemas de producción agrícola y tipologías en la agricultura hacen que estas zonas sean más vulnerables al efecto del cambio climático ya que, parte de la población depende de esta actividad para su subsistencia. Por otro lado, el planteamiento de estrategias que les permitan adaptarse a estos retos demuestra deficiencias, develando impactos negativos a escalas diferentes, en el ambiente y en la productividad de cultivos como el trigo o el arroz según las agroecologías (Jat et al., 2016).

Entre 1976 y 1989, fotografías permitieron identificar los cambios del uso de tierra y las variaciones climáticas producidas en los Andes (Vanacker et al., 2003); y que requirieron la reconformación de cobertura vegetal para reducir la pérdida de tierras de cultivo, suelos, insectos, malezas y ganado (Erda, 1996). Por ejemplo, la población de Guangaje en Ecuador es muy vulnerable a los cambios cíclicos en su producción agrícola generando escenarios de inseguridad alimentaria por períodos estacionales lo cual explicaría los altos niveles de desnutrición en esta población. Ante esta problemática (Blackmore et al., 2021) plantearon las siguientes soluciones: ampliación de la diversidad de cultivos, sistemas de retención de agua y riego para volverlos más resistentes al clima y garantizar la seguridad alimentaria de los distintos medios de vida.

Tabla 1

Investigaciones realizadas en cuerpos de agua por períodos geológicos

\begin{tabular}{|c|c|c|c|c|c|c|}
\hline \multirow{2}{*}{ Período } & \multirow{2}{*}{ País } & \multicolumn{2}{|c|}{ Coordenadas } & \multirow{2}{*}{ Variable climática } & \multirow{2}{*}{$\begin{array}{c}\text { Cuerpos de } \\
\text { agua }\end{array}$} & \multirow{2}{*}{ Referencia } \\
\hline & & Long. & Lat. & & & \\
\hline Holoceno & Colombia & $75^{\circ} \mathrm{W}$ & $5^{\circ} \mathrm{N}$ & Temperatura & Lagos & (Iriarte et al., 2020) \\
\hline Holoceno & Perú & $75^{\circ} \mathrm{O}$ & $15^{\circ} \mathrm{S}$ & $\begin{array}{l}\text { Temperatura } \\
\text { Precipitación }\end{array}$ & Ríos & (Viveen et al., 2019) \\
\hline Holoceno & Chile & $66^{\circ} \mathrm{W}$ & $24^{\circ} \mathrm{S}$ & Viento & Lagos & (Nanavati et al., 2019) \\
\hline Cuaternario & Argentina & $65^{\circ} \mathrm{W}$ & $20^{\circ} \mathrm{S}$ & Viento & Glaciar & (Gili et al., 2017) \\
\hline Holoceno & Venezuela & $73^{\circ} \mathrm{O}$ & $59^{\circ} \mathrm{N}$ & Temperatura & Lagos & (Stansell et al., 2014) \\
\hline
\end{tabular}


Tabla 2

Clasificación por autores de estudios sobre especies amenazadas

\begin{tabular}{|c|c|c|c|c|}
\hline País & Especie & Clasificación según el libro rojo & Altitud & Referencia \\
\hline Ecuador & $\begin{array}{l}\text { Aves frugívoras e insectívoras } \\
\text { Weinmannia }\end{array}$ & Vulnerable (VU) & 2500 & (Santillán et al., 2020) \\
\hline Argentina & $\begin{array}{l}\text { Madera } \\
\text { Jacaranda mimosifolia, } \\
\text { Handroanthus impetiginosus } \\
\text { Anadenanthera colubrina }\end{array}$ & $\begin{array}{l}\text { Vulnerable (VU) } \\
\text { Menor preocupación (LC) } \\
\text { Menor preocupación (LC) }\end{array}$ & 3000 & (Carón et al., 2018) \\
\hline $\begin{array}{l}\text { Argentina y } \\
\text { Chile }\end{array}$ & $\begin{array}{l}\text { Pleurodema thaul } \\
\text { Pleurodema bufoninum } \\
\text { Batrachyla taeniata }\end{array}$ & $\begin{array}{l}\text { Menor preocupación (LC) } \\
\text { Menor preocupación (LC) } \\
\text { Menor preocupación (LC) }\end{array}$ & 3000 & (Perotti et al., 2018) \\
\hline Brasil & $\begin{array}{l}\text { Primates } \\
\text { Papio hamadryas } \\
\text { Ateles geoffroyi } \\
\text { Alouatta pigra } \\
\text { Leontopithecus chrysomelas } \\
\text { Macaca sylvanus }\end{array}$ & $\begin{array}{l}\text { Menor preocupación (LC) } \\
\text { Vulnerable (VU) } \\
\text { En peligro de extinción (EN) } \\
\text { En peligro de extinción (EN) } \\
\text { En peligro de extinción (EN) }\end{array}$ & 500 & (Graham et al., 2016) \\
\hline
\end{tabular}

Las respuestas efectivas a estos impactos requieren conocer que las personas conceptualizan y experimentan los cambios ambientales de manera diferente y que esto puede influir en la cosmovisión local y los sistemas de valores de muchos grupos sociales (Scoville, 2018), permitiendo optimizar el uso de la tierra y su capacidad para adaptarse (Berrouet et al., 2020). A continuación, en la Tabla 3 se presentan estudios relacionados a la agricultura y cambio climático.

Estos estudios demuestran que, aunque el cambio climático parece ser un factor que influye en las prácticas de producción agrícola y ganadera, otras fuerzas no climáticas juegan un papel más importante en la determinación de su intensidad y adopción (López et al., 2017). Por ello, su planificación no solo debe basarse en escenarios futuros derivados de modelos numéricos, sino que también debe considerar las necesidades sociales, las agendas económicas, los conflictos políticos, la desigualdad socioeconómica y los valores culturales (Vuille et al., 2018). Por otro lado, en nuestro país, la gran capacidad de adaptación de quinua (Chenopodium quinoa Willd) facilitaría los retos que derivan del cambio climático pues su desarrollo no solo se da en regiones andinas sino también se ha expandido a zonas costeras. Lo que ha propiciado una creciente competencia por el uso de la tierra en muchas regiones (Bedoya et al., 2018) ya que se ha optado por reemplazar los procesos de rotación de cultivos por una agricultura intensiva que a largo plazo disminuirá la agrobiodiversidad de otros cultivos como la cañihua, tarwi o mashua.

\subsubsection{Erosión del suelo}

Estudiar cómo ocurre este proceso cerca de cuencas hidrográficas, ha permitido establecer que el cambio climático puede ayudarnos a comprender no solo cómo opera la evolución del paisaje, sino predecir sus consecuencias en la disponibilidad del recurso suelo. Es así que, Ochoa et al. (2016) analizaron si tanto el clima y la topografía influyen en esta variable planteando el método de estimación de riesgo, en el que las áreas protegidas con amplia cobertura vegetal tienen un mejor potencial para contrarrestar la erosión, a diferencia de zonas donde las pendientes son pronunciadas, hay escasez de cobertura vegetal y lluvias esporádicas pero de alta intensidad, que junto con la profundidad del suelo, incrementan el riesgo por erosión (Correa et al., 2016) representando un peligro latente para las poblaciones que desarrollan actividades agrícolas cerca de estas zonas. Por lo que, la distribución espacial de los bosques permitirá conocer los índices de deforestación y pérdida de cobertura vegetal (Bax \& Francesconi, 2018) evitando el incremento de estos peligros (Riquetti et al., 2020).

Esforzarse por alimentar a una población que llegará a casi 10 mil millones de personas en 2050 de forma sostenible es la prioridad de investigadores y políticos en la actualidad, ya que la mayor intensificación y expansión de las tierras agrícolas sería motivo de gran preocupación para el medio ambiente (Ortiz et al., 2021). En la Tabla 4 se presentan los principales aportes por autores respecto a este aspecto. 
Tabla 3

Autores que desarrollaron estudios sobre agricultura y cambio climático en los Andes

\begin{tabular}{|c|c|c|c|c|c|c|}
\hline \multirow{2}{*}{ País } & \multicolumn{2}{|c|}{ Coordenadas } & \multirow{2}{*}{ Altitud } & \multirow{2}{*}{ Cultivo } & \multirow{2}{*}{$\begin{array}{l}\text { Capacidad de } \\
\text { adaptación }\end{array}$} & \multirow{2}{*}{ Referencias } \\
\hline & Long. & Lat. & & & & \\
\hline Ecuador & $78^{\circ} \mathrm{W}$ & $2^{\circ} \mathrm{S}$ & 3500 & Mashua & Negativo & (Blackmore et al., 2021) \\
\hline Colombia & $74^{\circ} \mathrm{W}$ & $5^{\circ} \mathrm{N}$ & 1000 & Maíz & Positivo & (Iriarte et al., 2020) \\
\hline Colombia & $74^{\circ} \mathrm{W}$ & $5^{\circ} \mathrm{N}$ & 1000 & Рapa & Negativo & (Uribe et al., 2020) \\
\hline Perú & $75^{\circ} \mathrm{W}$ & $10^{\circ} \mathrm{S}$ & 4500 & Maíz & Positivo & (Zimmerer et al., 2019) \\
\hline Perú & $75^{\circ} \mathrm{W}$ & $10^{\circ} \mathrm{S}$ & 4500 & Рapa & Negativo & (Grados \& Schrevens, 2019) \\
\hline
\end{tabular}

En la literatura, se ha señalado la necesidad de cuantificar y caracterizar el capital natural del suelo bajo diferentes tipos de uso y cobertura (Machado et al., 2019). Así como, la identificación de las relaciones existentes entre la forma del paisaje, la erosión y el clima en regiones de rejuvenecimiento paisajístico, asociadas a una mayor denudación (Schlunegger \& Norton, 2013).

Del mismo modo, se ha visto el desarrollo de herramientas que permitan realizar una evaluación cuantitativa en los cambios pasados de este proceso en las cuencas hidrográficas (Dosseto \& Schaller, 2016). Aspectos que resultan clave para evitar la sobreexposición de las poblaciones andinas y la pérdida de grandes hectáreas de cultivos.

\subsubsection{Retroceso de glaciares}

El cambio ambiental moderno en los Andes probablemente se plasme mejor en el rápido derretimiento de los glaciares durante las últimas décadas (Michelutti et al., 2015); pues (Vargo et al., 2018) probaron la influencia de los impulsores climáticos como: altitud, temperatura y radiación en la sensibilidad glaciar de tres regiones de los Andes subtropicales demostrando que su disminución influye en zonas húmedas y áridas (Martini \& Astini, 2019). Asimismo, los lagos que son alimentados por estos glaciares constituyen importantes indicadores del cambio climático y su abundancia permite el establecimiento de redes de monitoreo garantizando el suministro de agua y energía de las poblaciones locales, entre las que destacan tres capitales: Lima (Perú), La Paz (Bolivia) y Quito (Ecuador) (Vuille et al., 2018). Es así como, la sensibilidad de los sistemas agrícolas ha ido en aumento en los últimos años requiriendo un mayor énfasis en el efecto que tienen el calor y agua en su rendimiento dejando de lado los efectos producidos por los cambios climatológicos intraestacionales de los Andes (Ponce, 2020).

Por otro lado, la disminución del volumen de los glaciares afecta el servicio brindado por las cuencas de montaña (Kinouchi et al., 2019) ya que sus superficies de hielo en glaciares y el subsuelo de hielo en el permafrost constituyen importantes torres de agua que servirán como reservas de este recurso para el futuro (Haeberli \& Weingartner, 2020). Al desaparecer por efecto del calentamiento global causarán que el agua en algunas regiones se incremente de manera temporal (Vuille et al., 2018) y en otras se agote provocando que impactos ambientales sean inevitables e irreversibles. Ante esto, Seo (2011) analizó los mecanismos de gestión del agua, tanto público como privado, en 7 países de América del Sur y sus estrategias en el sistema agrícola para afrontar el cambio climático. Demostró que, en períodos de escasez e incremento de la temperatura local, el sistema público brinda mejores posibilidades de acceso al agua sin comprometer su normal abastecimiento. Por otro lado, el riego privado se da de manera paulatina propiciando demasiada o poca provisión, lo cual no resultaría conveniente.

\subsubsection{Incremento de la susceptibilidad de ecosistemas de montaña}

Las diferentes proyecciones sugieren que es probable que la tasa de calentamiento en la troposfera inferior aumente con la altitud, impactando las montañas altas en gran parte de América del Sur y que al ser ejecutados con una resolución demasiado burda produzcan un suavizado de los gradientes de precipitación y temperatura local que son importantes para muchos procesos hidrológicos (Kinouchi et al., 2019). Cabe resaltar, que las montañas son las más susceptibles al cambio climático y la escasez de datos meteorológicos limita la comprobación de esta información; por lo que (Bennett et al., 2016) analizaron estos registros, con el fin de explicar y analizar este mecanismo en zonas secas y compararlo con zonas húmedas. La interacción entre la circulación atmosférica y la compleja topografía de la cordillera de los Andes tiene una fuerte influencia en el clima regional haciéndola más vulnerable. En la Tabla 5 se presenta la susceptibilidad de diferentes ecosistemas de montaña.

Parámetros topográficos como la ubicación geográfica (Buytaert et al., 2006) pueden servir como indicadores de cambios en la precipitación porque constituyen una importante fuente de agua para valles cercanos. Este es el caso de Bolivia que se caracteriza por sus altos niveles de pobreza y desigualdad, así como su situación topográfica y que es afectado por las continuas reducciones en el suministro de agua por el cambio climático (Kinouchi et al., 2019). Así, los agricultores de la zona han optado por intercalar y diversificar sus cultivos para mejorar su capacidad de resiliencia ante la problemática actual.

\subsection{Efectos en la agricultura de la zona costera}

La productividad agrícola y su efectividad para adaptarse a escenarios climáticos adversos requieren de más información sobre los riesgos que estos puedan causar (Laudien et al., 2020). Actualmente, algunas medidas de mitigación se asocian al recurso hídrico garantizando su protección ante condiciones climáticas secas basándose en modelos estadísticos y algoritmos de aprendizaje automáticos.

A nivel de Latinoamérica, los riesgos de inundación han aumentado en los últimos años, requiriendo la construcción de diques para minimizar los impactos en los cultivos representativos como: el azúcar, arroz, cebada, papa y trigo; pero que no garantizan su preservación a largo plazo, según indicó la Organización de las Naciones Unidas para la Alimentación y la Agricultura (FAO). 
Tabla 4

Clasificación por autores sobre estudios de erosión de suelos en los Andes

\begin{tabular}{|c|c|c|c|c|}
\hline País & Altitud & $\begin{array}{l}\text { Índice de } \\
\text { erosión }\end{array}$ & Clasificación & Referencia \\
\hline Perú & 4000 & 3 & Alto & (Rosas y Gutierrez, 2020) \\
\hline Colombia & 2100 & 2 & Medio & (Guzman et al., 2019) \\
\hline Colombia & 2100 & 2 & Medio & (Machado et al., 2019) \\
\hline Ecuador & 2200 & 2 & Medio & (Ochoa et al., 2016) \\
\hline
\end{tabular}

Uno de estos países es Colombia, cuyo aporte en el sector alimentario está posicionándose tanto en importación como exportación de mango y arroz pero que se ha visto afectado por estos eventos climáticos lo que ha causado que requiera medidas urgentes de adaptación optando por soluciones provisionales. Mientras que, en Venezuela, Argentina y Chile las inundaciones o sequías se dan a pasos agigantados permitiendo incluso pronosticar que las actividades agrícolas desparecerán por completo en unos años (Villanueva, 2005).

En el caso del Perú, muchas de las poblaciones andinas migraron hacia áridos valles, pues la abundancia de especies marítimas propicia un mejor desarrollo agrícola mediante la técnica de terrazas elevadas en suelos cultivables de grano fino. Este desarrollo se dio de manera simultánea a los cambios ambientales naturales generados por el incremento de la descarga de agua y sedimentos (Goodbred et al., 2020). Por ejemplo, el proyecto especial Chavimochic, desarrollado en la región de la Libertad, fue diseñado para el aprovechamiento del potencial hídrico del Río Santa en la generación de energía eléctrica, y que fue afectado por actividades mineras dañando su alta variedad de flora y fauna. Sumado a esto, el acelerado proceso de desglaciación por el incremento de la temperatura local puso en riesgo la oferta proyectada (Villanueva, 2005) requiriendo que se desvíe el agua del río para continuar las actividades agrícolas pero que trajo consigo la disminución del flujo de corriente, conflictos sociales, modificación del uso de la tierra y la búsqueda de nuevas herramientas tecnológicas que permitan abastecer de nuevo a las poblaciones con este recurso (Carey et al., 2014).

Otros casos registrados tuvieron lugar en los distritos de Santiago de Anchucaya, Huarochirí y Sangallaya en los que la escasez de agua hizo necesaria la construcción de la represa Huilco y la recuperación de 25 km del canal Julio C. Tello con la finalidad de garantizar el normal desarrollo de la actividad agrícola. Asimismo, en el departamento de Piura, una zona altamente productiva fue perjudicada por la poca disponibilidad del recurso hídrico debido al incremento de la temperatura en la zona y eventos climáticos extremos como el fenómeno del niño que a futuro desabastecerán de alimentos primordiales como el arroz, mango, limón, etc. (Scoville, 2018).

En todos los casos, la proyección que se tiene respecto a la necesidad de implementar infraestructuras de riego como mecanismo de respuesta ante el cambio climático, es que serán afectadas por el incremento de carga orgá- nica y a medida que se acelere el retroceso de los glaciares, cambiará la hidrología de los ríos, la demanda de agua aumentará, la contaminación empeorará y la competencia por el agua entre los sectores económicos, las jurisdicciones políticas y los usuarios del agua río arriba y río abajo se intensificarán (Lynch, 2012) requiriéndose mejoras en las políticas públicas para una mejor administración. Ante esta situación, el uso del agua no es predecible si solo es enfocado desde su disponibilidad. Por lo que, deberá considerarse la escorrentía de glaciares relacionados a modelos de almacenamiento y que la alteración humana de la dinámica en estas cuencas puede regirse por otros factores legales, económicos, políticos, culturales y sociales que realmente dan forma a los patrones de uso del agua y que resultan importantes en la formulación de planes de adaptación al cambio climático que garanticen la seguridad alimentaria (Carey et al., 2014).

\subsection{Efectos en la agricultura de otras zonas}

A diferencia de Latinoamérica, en otros países se aplicaron metodologías de evaluación del índice de vulnerabilidad de los sistemas agrícolas al cambio climático, uno de ellos fue el Estado de Karnataka en Canadá para lo cual recopilaron datos de pérdidas de producción de cultivos, exposición, sensibilidad y capacidad de adaptación donde asignaron ponderaciones a cada indicador logrando priorizar los distritos más vulnerables, con el fin de aplicar medidas de mitigación a cultivos de cereales, legumbres y oleaginosas. También estimaron que, alrededor del 70\% de la superficie cultivada se enfrenta a un nivel de vulnerabilidad de "extremo a alto" proponiendo la implementación de sitios específicos como mejores instalaciones de salud y educación, expansión de oportunidades de empleo en otros sectores o reducir la dependencia excesiva de la agricultura (Kumar et al., 2016). Mientras que en la región rural de Le Marche (Italia) la actividad agrícola arable cumple un rol importante en el desarrollo económico de la zona. Sin embargo, este sistema se ha visto afectado por el uso de sustancias contaminantes y los efectos ambientales negativos provocados por el cambio climático. Por ello, buscaron analizar si es aplicable el concepto de ecoeficiencia a una muestra de explotaciones agrícolas y evaluaron mediante la ponderación de dos metodologías cuál de estos sistemas era óptimo. Además, de indicar que estas explotaciones agrícolas son más ecoeficientes si están dirigidas por jóvenes agricultores que se encuentran participando en planes agroambientales (Bonfiglio et al., 2017). 
Tabla 5

Clasificación de susceptibilidad de ecosistemas de montaña

\begin{tabular}{|c|c|c|c|c|c|c|}
\hline \multirow[b]{2}{*}{ País } & \multicolumn{2}{|c|}{ Coordenadas } & \multirow[b]{2}{*}{ Alt. } & \multirow{2}{*}{$\begin{array}{l}\text { Clasificación según } \\
\text { altitud }\end{array}$} & \multirow{2}{*}{$\begin{array}{c}\text { Pisos } \\
\text { térmicos }\end{array}$} & \multirow{2}{*}{ Referencia } \\
\hline & Long. & Lat. & & & & \\
\hline Argentina & $66^{\circ} \mathrm{W}$ & $24^{\circ} \mathrm{S}$ & 6000 & Muy grande altura & Glacial & (Riquetti et al., 2020) \\
\hline Bolivia & $70^{\circ} \mathrm{W}$ & $20^{\circ} \mathrm{S}$ & 4000 & Gran Altura & Glacial & \\
\hline Chile & $68^{\circ} \mathrm{W}$ & $24^{\circ} \mathrm{S}$ & 3750 & Gran Altura & Páramo & (Martini \& Astini, 2019) \\
\hline Argentina & $66^{\circ} \mathrm{W}$ & $24^{\circ} \mathrm{S}$ & 6000 & Muy grande altura & Glacial & \\
\hline Ecuador & $78^{\circ} \mathrm{W}$ & $2^{\circ} \mathrm{S}$ & 3500 & Gran Altura & Páramo & (Pineda \& Willems, 2018) \\
\hline Perú & $75^{\circ} \mathrm{W}$ & $15^{\circ} \mathrm{S}$ & 4323 & Gran Altura & Glacial & (Michelutti et al., 2015) \\
\hline Bolivia & $70^{\circ} \mathrm{W}$ & $20^{\circ} \mathrm{S}$ & 3500 & Gran Altura & Glacial & (Rangecroft et al., 2013) \\
\hline Chile & $71^{\circ} \mathrm{W}$ & $30^{\circ} \mathrm{S}$ & 3750 & Gran Altura & Páramo & (Souvignet \& Heinrich, 2011) \\
\hline
\end{tabular}

Similares metodologías fueron aplicadas por otros países miembros de la Unión Europea como Alemania, Suecia o Austria, que a pesar de contar con estrictos estándares de protección ambiental no alcanzarían el nivel de eficiencia de países como Dinamarca, Bélgica, España, Francia o Irlanda (Vlontzos et al., 2014). Por lo que, el crecimiento de la productividad agrícola es vital para lograr mejores resultados económicos y de seguridad alimentaria que se ven amenazados por el cambio climático. En respuesta, los gobiernos y las agencias de desarrollo están fomentando la adopción de tecnologías agrícolas "climáticamente inteligentes" (Michler et al., 2019) para hacerle frente a esta problemática ambiental.

\section{Conclusiones}

Existe evidencia suficiente para afirmar que, a lo largo de la historia de la tierra, los factores climáticos como, precipitación, humedad y temperatura, aceleraron el proceso de desglaciación en los Andes, influyendo en la vulnerabilidad actual de los sistemas agrícolas al cambio climático. Esto provocará que países como, Brasil, Bolivia, Ecuador, Venezuela, Guyana y Colombia, incrementen su temperatura local, potencial de evapotranspiración y escasez de agua generando la pérdida de cultivos importantes como el arroz. En contraste, países como, Perú, Argentina, Chile, Bolivia y Uruguay registrarán temperaturas más bajas que afectarán su producción y rendimiento en cultivos como la quinua, papa, tarwi, entre otros cultivos.

Asimismo, la vulnerabilidad y distribución de especies de flora y fauna de los Andes ha sido ampliamente estudiada y asociada al uso de la tierra; pero que en ciertas zonas se ha limitado su normal desarrollo en todas sus etapas reproductivas y que dificultan su conservación, por lo que se recomendaría intensificar el uso de nuevas tecnologías de subsistencia

También, deberá priorizarse la creación de instituciones que se dediquen a temas de investigación de sistemas agrícolas presentes en los Andes porque garantizarían una mayor diversidad genética de cultivos haciéndolas capaces de resistir a plagas y enfermedades, mediante el establecimiento de enfoques orientados a la conservación de recursos genéticos almacenados en el germoplasma y su posterior registro, según las preferencias y estrategias de siembra en estas zonas.

Finalmente, se hace necesaria la implementación de metodologías que permitan evaluar el índice de vulnerabilidad de los sistemas agrícolas, que ofrecen grandes ventajas a la hora de priorizar zonas que son altamente vulnerables a los impactos del cambio climático porque permiten establecer medidas más puntuales según el escenario agroclimático a evaluarse.

\section{ORCID}

A. Lozano-Povis (D) https://orcid.org/0000-0002-6748-4367

C. E. Alvarez-Montalván (D) https://orcid.org/0000-0001-9110-8006

N. Moggiano-Aburto (D) https://orcid.org/0000-0002-5642-3833

\section{Referencias bibliográficas}

Agudelo, J., Urbina, N., \& Armenteras, D. (2019). Critical shifts on spatial traits and the risk of extinction of Andean anurans: an assessment of the combined effects of climate and land-use change in Colombia. Perspectives in Ecology and Conservation, 17(4), 206-219.

Bax, V., \& Francesconi, W. (2018). Environmental predictors of forest change: An analysis of natural predisposition to deforestation in the tropical Andes region, Peru. Applied Geography, 97, 99-110.

Bedoya, N., Pumi, G., Talamini, E., et al. (2018). The quinoa boom in Peru: Will land competition threaten sustainability in one of the cradles of agriculture? Land Use Policy, 79, 475-480

Bennett, M., New, M., Marino, J., \& Sillero-Zubiri, C. (2016). Climate complexity in the Central Andes: A study case on empirically-based local variations in the Dry Puna. Journal of Arid Environments, 128,40-49.

Berrouet, L., Villegas, C., \& Botero, V. (2020). Vulnerability of Rural Communities to Change in an Ecosystem Service Provision: Surface water supply. A Case Study in the Northern Andes, Colombia. Land Use Policy, 97, 104737

Blackmore, I., Rivera, C., Waters, W. , et al. (2021). The Impact of Seasonality and Climate Variability on Livelihood Security in the Ecuadorian Andes. Climate Risk Management, 32, 100279

Bonfiglio, A., Arzeni, A., \& Bodini, A. (2017). Assessing eco-efficiency of arable farms in rural areas. Agricultural Systems, 151, 114-125.

Bonnesoeur, V., Locatelli, B., Guariguata, M., et al. (2019). Impacts of forests and forestation on hydrological services in the Andes: A systematic review. In Forest Ecology and Management, 433, 569-584

Butt, N., \& Gallagher, R. (2018). Using species traits to guide conservation actions under climate change. Climatic Change, 151(2), 317-332.

Buytaert, W., Celleri, R., Willems, P., et al. (2006). Spatial and temporal rainfal variability in mountainous areas: A case study from the south Ecuadorian Andes. Journal of Hydrology, 329(3-4), 413-421.

Carey, M., Baraer, M., Mark, B., et al. (2014). Toward hydro-social modeling: Merging human variables and the social sciences with climate-glacier runoff models (Santa River, Peru). Journal of Hydrology, 518, 60-70.

Carón, M., De Frenne, P., Ortega, P., et al. (2018). Regeneration responses to climate and land-use change of four subtropical tree species of the southern Central Andes. Forest Ecology and Management, 417, 110-121.

Clerici, N., Cote, F., Escobedo, F., et al. (2019). Spatio-temporal and cumulative effects of land use-land cover and climate change on two ecosystem services in the Colombian Andes. Science of the Total Environment, 685, 1181-1192.

Correa, S., Mello, C., Chou, S., et al.(2016). Soil erosion risk associated with climate change at Mantaro River basin, Peruvian Andes. CATENA, 147, $110-$ 124

Dosseto, A., \& Schaller, M. (2016). The erosion response to Quaternary climate change quantified using uranium isotopes and in situ-produced cosmogenic nuclides. Earth-Science Reviews, 155, 60-81.

Engel, Z., Skrzypek, G., Chuman, T., et al. (2014). Climate in the Western Cordillera of the Central Andes over the last 4300 years. Quaternary Science Reviews, 99, 60-77.

Erda, L. (1996). Agricultural vulnerability and adaptation to global warming in China. Water, Air, and Soil Pollution, 92(1-2), 63-73. 
Fritz, S., Baker, P., Ekdahl, E., et al. (2010). Millennial-scale climate variability during the Last Glacial period in the tropical Andes. Quaternary Science Reviews, 29(7-8), 1017-1024.

Gili, S., Gaiero, D., Goldstein, S., et al. (2017). Glacial/interglacial changes of Southern Hemisphere wind circulation from the geochemistry of South American dust. Earth and Planetary Science Letters, 46, 98-109.

Goodbred, S., Dillehay, T., Galvéz, C., et al. (2020). Transformation of maritime desert to an agricultural center: Holocene environmental change and landscape engineering in Chicama River valley, northern Peru coast. Quaternary Science Reviews, 22, 1-13.

Gorostiague, P., Sajama, J., \& Ortega, P. (2018). Will climate change cause spatial mismatch between plants and their pollinators? A test using Andean cactus species. Biological Conservation, 226, 247-255.

Grados, D., \& Schrevens, E. (2019). Multidimensional analysis of environmental impacts from potato agricultural production in the Peruvian Central Andes. Science of the Total Environment, 663, 927-934.

Graham, T., Matthews, H., \& Turner, S. (2016). A Global-Scale Evaluation of Primate Exposure and Vulnerability to Climate Change. International Journal of Primatology, 37(2), 158-174.

Guzman, C., Hoyos, F., Da Silva, M., et al. (2019). Variability of soil surface characteristics in a mountainous watershed in Valle del Cauca, Colombia: Implications for runoff, erosion, and conservation. Journal of Hydrology, 576(2019), 273-286

Haeberli, W., \& Weingartner, R. (2020). In full transition: Key impacts of vanishing mountain ice on water-security at local to global scales. Water Security, 1, 100074

Iriarte, J., Elliott, S., Maezumi, S., et al. (2020). The origins of Amazonian landscapes: Plant cultivation, domestication and the spread of food production in tropical South America. Quaternary Science Reviews, 248, 106582.

Jara, I., Moreno, P., Alloway, B., et al (2019). A 15,400-year long record of vegetation, fire-regime, and climate changes from the northern Patagonian Andes. Quaternary Science Reviews, 226, 106005.

Jat, M., Dagar, J. , Sapkota, T., et al. (2016). Climate change and agriculture: Adaptation strategies and mitigation opportunities for food security in South Asia and Latin America. In Advances in Agronomy, 137,127-235.

Kinouchi, T., Nakajima, T., Mendoza, J., et al. (2019). Water security in high mountain cities of the Andes under a growing population and climate change: A case study of La Paz and El Alto, Bolivia. Water Security, 6 , 100025.

Kumar, S., Raizada, A., Biswas, H., et al. (2016). Application of indicators for identifying climate change vulnerable areas in semi-arid regions of India. Ecological Indicators, 70(2016), 507-517.

Laudien, R., Schauberger, B., Gleixner, S., et al. (2020). Assessment of weatheryield relations of starchy maize at different scales in Peru to support the NDC implementation. Agricultural and Forest Meteorology, 295, 108154.

López, S., Jung, J., \& López, M. (2017). A hybrid-epistemological approach to climate change research: Linking scientific and smallholder knowledge systems in the Ecuadorian Andes. Anthropocene, 17, 30-45.

Lüning, S., Gałka, M., Bamonte, F., et al. (2019). The Medieval Climate Anomaly in South America. Quaternary International, 508, 70-87.

Lynch, B. (2012). Vulnerabilities, competition and rights in a context of climate change toward equitable water governance in Peru's Rio Santa Valley. Global Environmental Change, 22(2), 364-373.

Machado, J., Villegas, C., Loaiza, J., et al. (2019). Soil natural capital vulnerability to environmental change. A regional scale approach for tropical soils in the Colombian Andes. Ecological Indicators, 96(65), 116-126.

Martini, M., \& Astini, R. (2019). Comment on "Sensitivity of glaciation in the arid subtropical Andes to changes in temperature, precipitation, and solar radiation" by Vargo et al. (2018). Global and Planetary Change, 172, 475478

Michelutti, N., Cooke, C., Hobbs, W., \& Smol, J. (2015). Climate-driven changes in lakes from the Peruvian Andes. Journal of Paleolimnology, 54(1), 153-160.

Michler, J., Baylis, K., Arends, M., et al. (2019). Conservation agriculture and climate resilience. Journal of Environmental Economics and Management, 93, 148-169.

Nanavati, W., Whitlock, C., Iglesias, V., et al. (2019). Postglacial vegetation, fire, and climate history along the eastern Andes, Argentina and Chile (lat. 41$\left.55^{\circ} \mathrm{S}\right)$. Quaternary Science Reviews, 20, 145-160.

Ochoa, P., Fries, A., Mejía, D., et al. (2016). Effects of climate, land cover and topography on soil erosion risk in a semiarid basin of the Andes. Catena $140,31-42$

Ortiz, A., Outhwaite, C., Dalin, C., et al. (2021). A review of the interactions between biodiversity, agriculture, climate change, and international trade: research and policy priorities. In One Earth 4(1), 88-101.

Perotti, M., Bonino, M., Ferraro, D., et al. (2018). How sensitive are temperate tadpoles to climate change? The use of thermal physiology and niche model tools to assess vulnerability. Zoology, 127, 95-105.
Pineda, L., \& Willems, P. (2018). Rainfall extremes, weather and climate drivers in complex terrain: A data-driven approach based on signal enhancement methods and EV modeling. Journal of Hydrology, 563, 283-302.

Ponce, C. (2020). Intra-seasonal climate variability and crop diversification strategies in the Peruvian Andes: A word of caution on the sustainability of adaptation to climate change. World Development, 127, 1-22.

Ramirez, J., Cuesta, F., Devenish, C., et al. (2014). Using species distributions models for designing conservation strategies of Tropical Andean biodiversity under climate change. Journal for Nature Conservation, 22(5), 391--404.

Rangecroft, S., Harrison, S., Anderson, K., et al. (2013). Climate change and water resources in arid mountains: An example from the bolivian andes. Ambio, 42(7), 852-863.

Ríos, C., Watson, J., \& Butt, N. (2018). Persistence of methodological, taxonomical, and geographical bias in assessments of species' vulnerability to climate change: A review. Global Ecology and Conservation, 15, 1-15.

Riquetti, N., Mello, C., Beskow, S., et al. (2020). Rainfall erosivity in South America: Current patterns and future perspectives. Science of the Total Environment, 724, 138315

Rosas,M., \& Gutierrez, R. (2020). Assessing soil erosion risk at national scale in developing countries: The technical challenges, a proposed methodology, and a case history. Science of the Total Environment, 703, 135474

Santillán, V., Quitián, M., Tinoco, B., et al. (2020). Direct and indirect effects of elevation, climate and vegetation structure on bird communities on a tropical mountain. Acta Oecologica, 102, 103500

Schlunegger, F., \& Norton, K. (2013). Water versus ice: The competing roles of modern climate and Pleistocene glacial erosion in the Central Alps of Switzerland. Tectonophysics, 602, 370-381.

Scoville, M. (2018). Climate, the Earth, and God - Entangled narratives of cultural and climatic change in the Peruvian Andes. World Development, $170,345-359$

Seo, S. (2011). An analysis of public adaptation to climate change using agricultural water schemes in South America. Ecological Economics, 70(4), 825-834.

Souvignet, M., \& Heinrich, J. (2011). Statistical downscaling in the arid central Andes: Uncertainty analysis of multi-model simulated temperature and precipitation. Theoretical and Applied Climatology, 106(1-2), 229-244.

Stansell, N., Polissar, P., Abbott, M., et al. (2014). Proglacial lake sediment records reveal Holocene climate changes in the Venezuelan Andes. Quaternary Science Reviews, 89, 44-55.

Trauth, M., Bookhagen, B., Marwan, N., et al. (2003). Multiple landslide clusters record Quaternary climate changes in the northwestern Argentine Andes. Palaeogeography, Palaeoclimatology, Palaeoecology, 194(1-3), 109-121.

Tuberville, T., Andrews, K., Sperry, J. H., et al. (2015). Use of the NatureServe Climate Change Vulnerability Index as an Assessment Tool for Reptiles and Amphibians: Lessons Learned. Environmental Management, 56(4), 822834.

Uribe, N., Srinivasan, R., Corzo, G., et al. (2020). Spatio-temporal critical source area patterns of runoff pollution from agricultural practices in the Colombian Andes. Ecological Engineering, 149, 105810.

Vanacker, V., Govers, G., Poesen, J., et al. (2003). The impact of environmental change on the intensity and spatial pattern of water erosion in a semi-arid mountainous Andean environment. Catena, 51(3-4), 329-347.

Vargo, L., Galewsky, J., Rupper, S., et al. (2018). Sensitivity of glaciation in the arid subtropical Andes to changes in temperature, precipitation, and solar radiation. Global and Planetary Change, 163, 86-96

Vicenzi, N.. Corbalán, V., Miles, D., et al. (2017). Range increment or range detriment? Predicting potential changes in distribution caused by climate change for the endemic high-Andean lizard Phymaturus palluma. Biological Conservation, 206, 151-160.

Villanueva, R. (2005). Características de la Cuenca del Río Santa. 37, 59-64.

Viveen, W., Zevallos, L., \& Sanjurjo, J. (2019). The influence of centennial-scale variations in the South American summer monsoon and base-level fall on Holocene fluvial systems in the Peruvian Andes. Global and Planetary Change, 176, 1-22.

Vlontzos, G., Niavis, S., Manos, B. (2014). A DEA approach for estimating the agricultural energy and environmental efficiency of EU countries. Renewable and Sustainable Energy Reviews, 40, 91-96.

Vuille, M., Carey, M., Huggel, C., et al. (2018). Rapid decline of snow and ice in the tropical Andes - Impacts, uncertainties and challenges ahead. EarthScience Reviews, 176, 195-213.

Williams, J., Gosling, W., Brooks, S., et al. (2011). Vegetation, climate and fire in the eastern Andes (Bolivia) during the last 18,000years. Palaeogeography, Palaeoclimatology, Palaeoecology, 312(1-2), 115-126.

Zimmerer, K., de Haan, S., Jones, A., Creed, H., et al. (2019). The biodiversity of food and agriculture (Agrobiodiversity) in the anthropocene: Research advances and conceptual framework. Anthropocene, 25, 100192. 